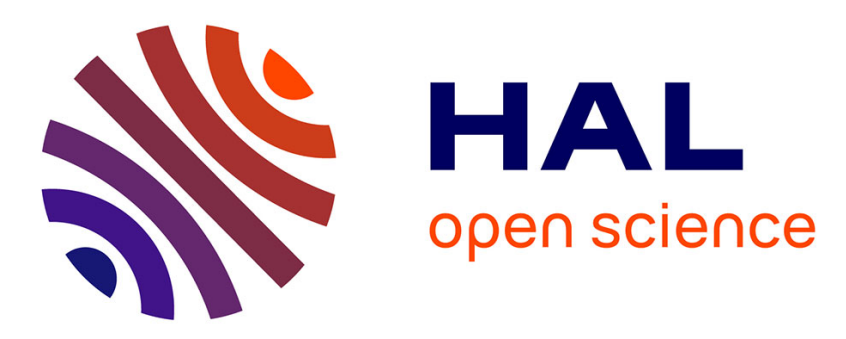

\title{
Influence of the filling factor on the spectral properties of plasmonic crystals
}

\author{
Anne Laure Baudrion, Jean Claude Weeber, Alain Dereux, Guillaume \\ Lecamp, Philippe Lalanne, S. I. Bozhevolnyi
}

\section{- To cite this version:}

Anne Laure Baudrion, Jean Claude Weeber, Alain Dereux, Guillaume Lecamp, Philippe Lalanne, et al. Influence of the filling factor on the spectral properties of plasmonic crystals. Physical Review B: Condensed Matter and Materials Physics (1998-2015), 2006, 74 (12), pp.125406. 10.1103/PhysRevB.74.125406 . hal-00437242

\section{HAL Id: hal-00437242 \\ https://hal.science/hal-00437242}

Submitted on 11 Apr 2016

HAL is a multi-disciplinary open access archive for the deposit and dissemination of scientific research documents, whether they are published or not. The documents may come from teaching and research institutions in France or abroad, or from public or private research centers.
L'archive ouverte pluridisciplinaire HAL, est destinée au dépôt et à la diffusion de documents scientifiques de niveau recherche, publiés ou non, émanant des établissements d'enseignement et de recherche français ou étrangers, des laboratoires publics ou privés. 


\title{
Influence of the filling factor on the spectral properties of plasmonic crystals
}

\author{
A.-L. Baudrion, J.-C. Weeber, and A. Dereux \\ Laboratoire de Physique de l'Université de Bourgogne, UMR CNRS 5027, 9 avenue A. Savary, Boîte Postale 47870, \\ F-21078 Dijon, France \\ G. Lecamp and P. Lalanne \\ Laboratoire Charles Fabry de l'Institut d'Optique, Centre National de la Recherche Scientifique, 91403 Orsay Cedex, France
}

\author{
S. I. Bozhevolnyi \\ Department of Physics and Nanotechnology, Aalborg University, Skjernvej 4A, DK-9220 Aalborg East, Denmark
}

(Received 13 April 2006; revised manuscript received 30 June 2006; published 5 September 2006)

\begin{abstract}
Plasmonics crystals (PCs) comprised of finite-size triangular lattices of gold bumps deposited on a gold thin film are studied by means of a near-field optical microscope. The plasmonic crystals fabricated by electronbeam lithography are illuminated by an incident surface plasmon polariton excited in the Kretschmann-Raether configuration at the gold/air thin-film interface for incident free-space wavelengths in the range 740-820 $\mathrm{nm}$. Based on the measurement of the surface plasmon polariton (SPP) damping distance in the crystals, the existence of a band gap for an incident SPP traveling along the two symmetry axes $\Gamma M$ and $\Gamma K$ is demonstrated. By increasing the lateral size of the bumps, the influence of the filling factor on the PC spectral properties is investigated. We show that, for the two $\Gamma M$ and $\Gamma K$ axes, the central gap frequency depends only weakly on the filling factor, whereas we observed a neat band-gap broadening for increasing filling factors. The experimental results are found to be consistent with the PC band structures computed by means of the differential method. In particular, a good agreement between the experimental and theoretical central gap frequencies for the two $\Gamma M$ and $\Gamma K$ axes is obtained. Finally, we investigate the origin of the band gap in PCs by computing modal reflectivity (or in-plane reflectivity) of semi-infinitely extended crystals. From these calculations, we show that the SPP propagation through a PC is inhibited by distributed reflection and not by losses channels such as absorption or out-of-plane scattering.
\end{abstract}

DOI: 10.1103/PhysRevB.74.125406 PACS number(s): 73.20.Mf, 78.66.-w, 07.79.Fc, 42.70.Qs

\section{INTRODUCTION}

Surface plasmons polaritons (SPPs) are electromagnetic excitations that can propagate along metal/dielectric interfaces. ${ }^{1}$ If the interface is flat, SPPs cannot be excited by light incident from the dielectric medium since, at a given frequency, the SPP wave-vector component $\left(k_{\|}^{\mathrm{SPP}}\right)$ parallel to the interface along which it propagates exceeds the wavevector modulus of the radiative light traveling in the dielectric medium. Two standard techniques are used to match the parallel incident wave-vector $k_{\|}^{\text {inc }}$ with that of a SPP. First, the surface of the metal can be illuminated with an evanescent wave produced by total internal reflection, and second, the amount of momentum to be added to the incident wave vector to reach $k_{\|}^{\mathrm{SPP}}$ can be gained from a grating input coupler. ${ }^{2}$ Similarly, following the inverse optical path, a SPP incident on a grating generates a scattered wave with a parallel wave-vector component $k_{\|}^{d}$ verifying the momentum conservation law $\vec{k}_{\|}^{d}=\vec{k}_{\|}^{\mathrm{SPP}}+\vec{G}$, where $\vec{G}$ is a reciprocal-lattice vector of the grating output coupler. If $\left|\vec{k}_{\|}^{d}\right|$ is smaller than $\left|\vec{k}_{\|}^{\mathrm{SPP}}\right|$, the incident SPP is said to be scattered out-of-plane as the incident SPP is converted into photons. In the event of $\left|\vec{k}_{\|}^{d}\right|$ equal to $\left|\vec{k}_{\|}^{\mathrm{SPP}}\right|$, the incident SPP is scattered in-plane since the scattered wave is still a SPP. The SPP in-plane scattering is at the origin of the energy gaps that open up at the boundaries of the Brillouin zones in the dispersion curves of the SPP sustained by periodically modulated surfaces. ${ }^{3-5}$

These gaps have been exploited for the design of SPP Bragg mirrors for SPPs traveling along the surface of ex- tended thin films ${ }^{6}$ or SPP modes supported by thin metal strips. ${ }^{7,8}$ The existence of these energy gaps has also been demonstrated in the case of bigrating of surface defects. In particular, it has been shown experimentally ${ }^{4}$ and theoretically ${ }^{9,11}$ that full band gaps can be obtained for twodimensional (2D) gratings with a triangular lattice. The existence of such an absolute gap implies that, over a given frequency range, a thin-film SPP cannot propagate through the grating for all in-plane propagation directions. In analogy with 3D photonic crystals, ${ }^{12}$ it has been suggested to use these 2D gratings of surface defects for controlling SPP propagation. By means of a near-field optical microscope, the guiding of SPPs along a straight and bent line defect opened into so-called plasmonic crystals (PCs) has been reported. ${ }^{13-15}$ Recent numerical calculations based on the Green dyadic formalism have pointed out that the width and/or the central frequency of the gap obtained for PCs represented by a triangular lattice of finite height cylinders depend strongly on structural parameters such as the radius of the scatterers. ${ }^{16,17}$ Thus, it has been established that significant improvement of PC guiding performances can be expected if their geometrical parameters are correctly chosen. On the other hand, the optimum choice has not yet been clearly identified, partially because the reported studies of SPP propagation in PCs were so far either experimental ${ }^{13-15}$ or theoretical, ${ }^{16,17}$ a circumstance that made a direct comparison and analysis of the results obtained quite difficult.

The objective of this work is to investigate both experimentally and theoretically the influence of the scatterers size 

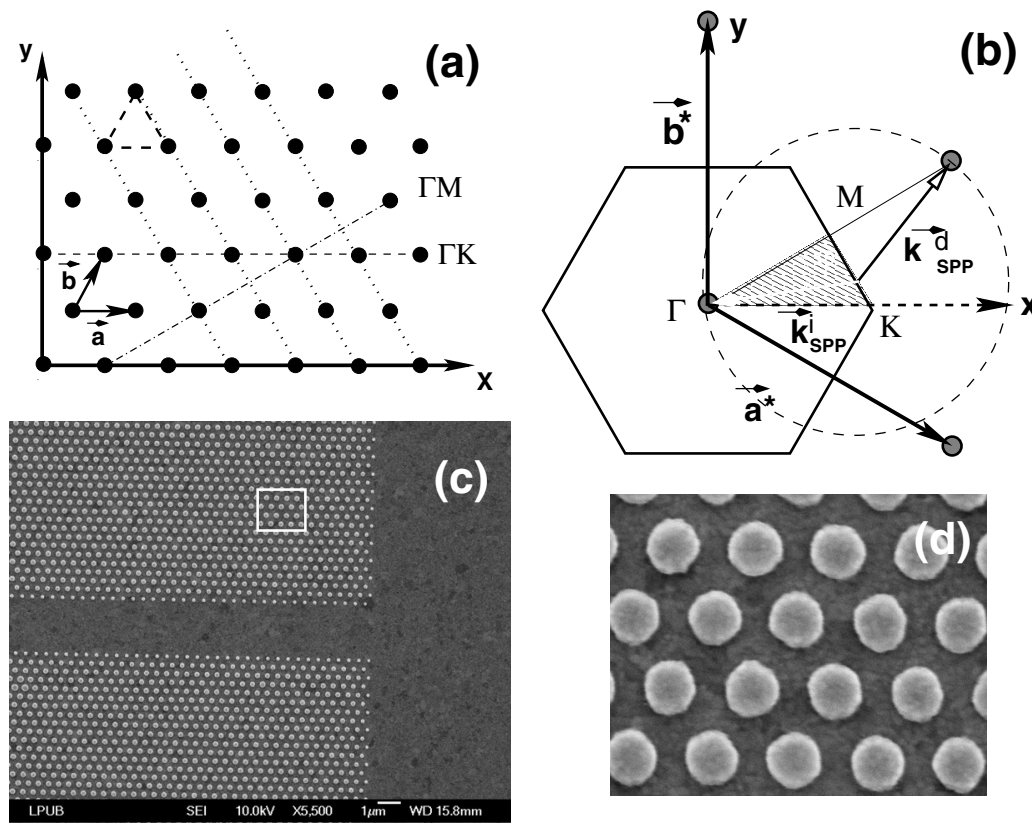

FIG. 1. (a) Direct space of a triangular lattice. The symmetry axes $\Gamma M$ and $\Gamma K$ are shown, respectively, as a dashed and a dash-dotted line. (b) First Brillouin zone of a triangular lattice. The irreducible Brillouin zone corresponds to the triangle $\Gamma K M$. (c) SEM images of a PBG structure consisting of a 430-nm-period triangular lattice of particles of 290-nm diameter. (d) Zoom of the PBG structure corresponding to the white square shown on image (c). (i.e., the filling factor) on the properties of PCs, including the SPP guiding along channels in PCs. Beyond the fundamental interest, knowing the influence of the filling factors on properties such as the central gap frequency or the width of the energy gaps is obviously highly desirable for the development of efficient PC-based integrated optical devices.

The paper is organized as follows. In Sec. II, we first describe the origin of the energy band gap in triangular gratings and we discuss the choice of the period of the samples we use in this work. In Sec. III, we briefly explain the fabrication method of the samples and we discuss more in detail the near-field optical characterization of the PC. Specifically, we consider the influence on the near-field images of a local or an extended illumination of the crystals. We present, in Sec. IV, the near-field images recorded at different frequencies over PC with increasing filling factors. On the basis of these images, we locate the energy gap for two in-plane propagation directions corresponding to the symmetry axes $\Gamma M$ and $\Gamma K$. In Sec. V, the experimental results are discussed and compared with numerical calculations performed with the differential method. Based on both numerical and experimental results, the influence of the filling factor on the PC properties is analyzed. Concluding remarks are given in Sec. VI.

\section{DIFFRACTION IN PLASMONIC CRYSTALS}

The samples we consider in this work consist of gold bumps deposited on a gold thin film and arranged to form a 2D triangular lattice (also called hexagonal lattice) with a period $a$. For the near-field optical imaging of these samples we use, a cw titanium-sapphire laser tunable from 740 to $830 \mathrm{~nm}$ as a light source. With the aim of observing the energy band gaps (BG) of the PC within the tunability range of our laser, the period of the grating must be chosen carefully. Figures 1(a) and 1(b) show, respectively, the direct and reciprocal space of a $2 \mathrm{D}$ triangular lattice. In direct space, the triangular lattice can be spanned using two vectors with components in the Cartesian basis given by $\vec{a}=(a, 0)$ and $\vec{b}=\left(\frac{a}{2}, \frac{\sqrt{3} a}{2}\right)$ such that the corresponding reciprocal vector components are $\vec{a}^{*}=\frac{2 \pi}{a}\left(1, \frac{-1}{\sqrt{3}}\right)$ and $\vec{b}^{*}=\frac{2 \pi}{a}\left(0, \frac{2}{\sqrt{3}}\right)$. From the basis vectors of the reciprocal lattice, the hexagonally shaped first Brillouin zone of the grating can be constructed. Taking into account the symmetry of this first Brillouin zone, the irreducible Brillouin zone defined by the symmetry points $\Gamma$, $M$, and $K$ is obtained. Note that our definition of the symmetry points $M\left(\frac{1}{2}, \frac{1}{2}\right)$ and $K\left(\frac{2}{3}, \frac{1}{3}\right)$ [coordinates given in the $\left(\vec{a}^{*}, \vec{b}^{*}\right)$ basis] that is the most widely accepted definition ${ }^{12}$ is, however, reversed compared to that of Refs. 13, 14, 16, and 17.

A complete study of the optical properties of the 2D triangular grating would require investigating the optical response of the PC to incident SPPs having an in-plane wave vector sweeping the boundary of the irreducible Brillouin zone. SPPs with in-plane wave vectors connecting $\Gamma$ to a point of the $M K$ segment intersect the boundary of the first Brillouin zone and then are Bragg reflected. Indeed, such SPPs are elastically scattered in the direction given by $\vec{k}_{\mathrm{SPP}}^{s}$ $=\vec{k}_{\mathrm{SPP}}^{i}+\vec{k}_{G}$, where $\vec{k}_{\mathrm{SPP}}^{i}$ denotes the incident SPP wave vector and where $\vec{k}_{G}$ is a Bragg vector of PC given by $\vec{k}_{G}=m \vec{a}^{*}$ $+n \vec{b}^{*}$, with $n$ and $m$ equal to $1,-1$, or 0 . Without any loss of generality, let us consider the irreducible Brillouin zone shown in Fig. 1(b). For this zone, the corresponding PC Bragg vector is given by $\vec{k}_{G}=\vec{a}^{*}+\vec{b}^{*}$. Thus, a SPP with a wave vector $\vec{k}_{\mathrm{SPP}}$ that belongs to this irreducible Brillouin zone will be Bragg reflected provided that

$$
\left|\vec{k}_{\mathrm{SPP}}\right| \cos \varphi=\frac{1}{2}\left|\vec{a}^{*}+\vec{b}^{*}\right|,
$$

where $\varphi$ is the angle formed by the propagation direction of the incident SPP and the symmetry axis $\Gamma M$. For the irreducible Brillouin zone considered in Fig. 1(b), the SPPs describ- 
ing the $M K$ segment are all Bragg reflected by the same crystal lattice lines with Miller indices equal to $(1,1)$ and shown in Fig. 1(a) as dotted lines. It is worth noting that a SPP traveling along the $\Gamma M$ direction is normally incident on the lattice lines that Bragg reflect it, while for any other propagation directions (on the $M K$ segment), the SPP hits these lines at oblique incidence. In particular, the propagation direction $\Gamma K$ corresponds to an angle of incidence on the $(1,1)$ lattice lines of $30^{\circ}$. It is then a simple matter to find from Eq. (1) the wave-vector modulus $k_{\mathrm{SPP}}=\frac{2 \pi}{\lambda_{\mathrm{SPP}}}$ of the SPPs that are Bragg reflected by the $\mathrm{PC}$ when propagating along a $\Gamma M$ or a $\Gamma K$ direction. Indeed, for a SPP traveling along the $\Gamma M$ direction, it is found that the Bragg reflection occurs provided that its wavelength $\lambda_{\mathrm{SPP}}$ verifies

$$
\lambda_{\mathrm{SPP}}=\sqrt{3} a,
$$

while for the $\Gamma K$ direction, the SPP should be Bragg reflected if

$$
\lambda_{\mathrm{SPP}}=\frac{3 a}{2} .
$$

It is clear that neither the exact central gap frequency nor the width of the gaps can be obtained from Eq. (1),, 3 as this reasoning holds only for crystals with a vanishing refractive index modulation, ${ }^{10}$ however these simple calculations provide us with, at least, a guideline for the design of the samples.

With the aim of centering the gap of at least one propagation direction with respect to our laser tunability range, we consider triangular lattices with a period $a=430 \mathrm{~nm}$. The corresponding Bragg reflected SPP wavelengths are, respectively, $\lambda_{\mathrm{SPP}}=744 \mathrm{~nm}$ for the $\Gamma M$ direction and $\lambda_{\mathrm{SPP}}$ $=645 \mathrm{~nm}$ for the $\Gamma K$ direction. The effective index of a SPP (defined as $n_{\mathrm{eff}}=\frac{\lambda_{0}}{\lambda_{\mathrm{SPP}}}$ ) traveling at the air/metal surface of a 50-nm-thick gold thin film deposited on a glass substrate has been computed from the dielectric function of gold tabulated in Ref. 18. From this effective index, we found that the center gap frequency for the $\Gamma M$ direction corresponds to a freespace wavelength of $\lambda_{0}=762 \mathrm{~nm}$ (effective index of the SPP $\left.n_{\text {eff }}=1.024\right)$ and to $\lambda_{0}=674 \mathrm{~nm}$ for the $\Gamma K$ direction $\left(n_{\text {eff }}\right.$ $=1.044)$. Thus, with such a grating period, the gap for the $\Gamma M$ direction should be centered with respect to the frequency range of our laser. Moreover, by using this period, we will possibly investigate the behavior of SPPs on the longwavelength edge of the gap for the $\Gamma K$ direction.

\section{NEAR-FIELD IMAGING}

The samples we consider in this study have been fabricated by standard electron-beam lithography. A 50-nm-thick gold film deposited on a glass substrate is first spin-coated with a 300-nm-thick layer of polymethylmethacrylate (PMMA). A field emission gun scanning electron microscope (SEM) controlled by a lithography system is used to create a triangular lattice of holes in the PMMA layer. A 50-nm-thick gold film is subsequently thermally evaporated onto the polymer and finally the dissolution of the nonexposed PMMA layer allows us to obtain a gold thin film textured by circular gold bumps of fixed height. The radius of the bumps is controlled by varying the dose during the exposure of the polymer. Figures 1(c) and 1(d) show SEM images of a typical sample. The samples consist of a textured area in which line-defect waveguides are opened. These line-defect waveguides can be oriented either along the $\Gamma M$ or the $\Gamma K$ directions. For the $\Gamma M$ direction, the channels are obtained by removing ten lines while only six lines are removed for the $\Gamma K$ orientation, such that in both cases the width of the channels is approximately $2.5 \mu \mathrm{m}$.

The interaction of the SPP with the PC is observed by means of a photon scanning tunneling microscope (PSTM). A detailed description of the experimental setup used in this work can be found in Ref. 19. The sample is glued with an index matching fluid to the hypotenuse of a right-angle glass prism comprising the stage of the PSTM. A TM-polarized titanium-sapphire laser is used to launch a surface plasmon polariton at the surface of the bare thin gold film in the Kretschmann-Raether configuration. A fine adjustment of the angle of incidence of the laser beam is performed by minimizing the power of the reflected beam. Note that with the surface occupied by the PC being negligible compared to that of the whole sample, we observe a dip in the reflectivity of the sample similar to that of a bare gold thin film. The plane of incidence of the incoming beam is oriented parallel to the long axis of the line defects opened in the PC. A multimode fiber tip produced by a standard heat-and-pull technique is subsequently coated with a metal multilayer comprised of $30 \mathrm{~nm}$ of chromium and $5 \mathrm{~nm}$ of gold (no resulting aperture at the end of the tip). The tip is attached to a piezotube and scanned at constant height in the very vicinity of the sample surface, allowing us to observe the near-field intensity distributions over the channels and the crystals.

Prior to discussing the properties of PC as a function of the filling factors, we first consider the influence of the illumination conditions on the near-field images of the channels. Most of the previous works dealing with PC have been performed by using a collimated beam rather that a focused one for the excitation of the SPP on the bare metal/air interface. Owing to the spot size used in these experiments, the line defects and the crystals are not only excited by the SPP launched on the bare thin film but also directly by the incident light. When using such an extended source of light illuminating the whole length of the channels, artifacts can occur that may lead to erroneous interpretations of the PC near-field images. In order to check the existence of such artifacts, we consider a $\Gamma M$ oriented channel opened in a crystal comprised of gold bumps with a radius of $70 \mathrm{~nm}$. Figures 2(a) and 2(b) show, respectively, the near-field images of this crystal when excited by using a collimated beam or a focused spot (spot radius about $5 \mu \mathrm{m}$ in the focal plane) with a frequency corresponding to a free-space wavelength of $\lambda_{0}=775 \mathrm{~nm}$. For the focused illumination, the incident spot is out of the scanning range of the image shown in Fig. 2(b) at a distance of about $10 \mu \mathrm{m}$ on the right border of this image. On the two PSTM images, the bare gold thin film is located on the right-hand side of the line defined by $x$ $=10 \mu \mathrm{m}$ and the SPP excited on this thin film travels from the right to the left of the images. A very neat standing-wave pattern created by the interference of the incident SPP with 

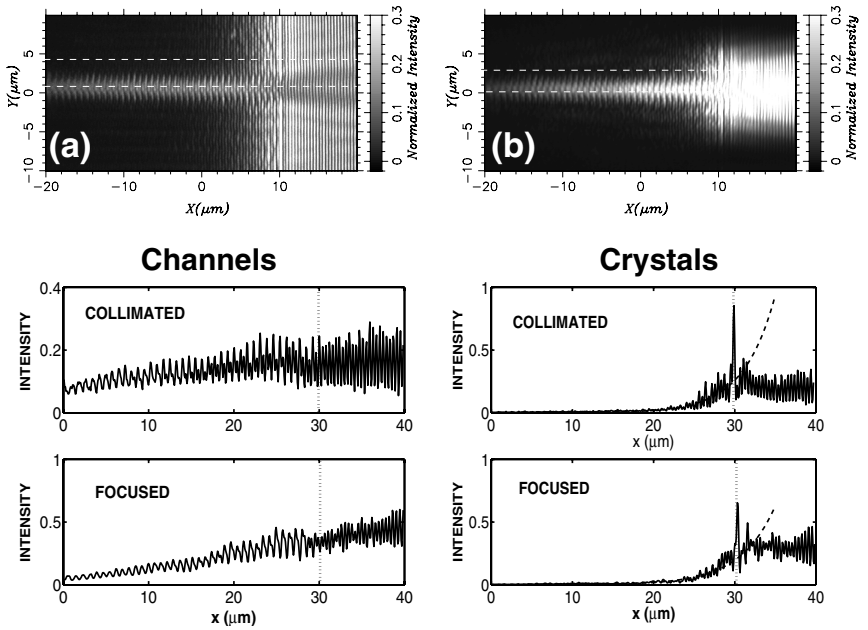

FIG. 2. Top: PSTM images of a $\Gamma M$ oriented PC obtained with, respectively, a collimated (a) or focused (b) illumination with an incident frequency corresponding to $\lambda_{0}=775 \mathrm{~nm}$. The overlaying white dashed lines locate the origin of the cross-cuts shown in the bottom of the figure. the incident thin-film SPP is launched on the thin film located on the right-hand side of the crystal $(x>10 \mu \mathrm{m})$ and travels from the right to the left of the images. Bottom: Crosscuts of the PSTM images taken along the channels (left) or over the crystals (right). The dotted lines on the cross-cuts show the boundary between the PC and the bare thin film. The inner crystal SPP damping distance is measured using the cross-cuts of the PSTM images taken over the crystal.

the SPP back-reflected by the crystal is visible on the right part of the two PSTM images. The intensity detected over the channel for both illuminations demonstrates that the incident SPP is efficiently guided or at least confined by the channel. In addition, it can be seen that the modal structures visible in the channel are qualitatively similar for the collimated and focused illuminations. Cross-cuts of the collimated and focused PSTM images taken over the channels are displayed in Fig. 2. It is worth noting that the near-field intensity modulation visible on the cross-cuts has a periodicity of roughly $385 \mathrm{~nm}$ outside the crystal and about $750 \mathrm{~nm}$ inside the $\Gamma M$ oriented channel. The reason for this difference can be attributed to the fact that outside the crystal, the standing wave pattern arises from the interference of two surface waves leading to a periodicity of $\lambda_{\mathrm{SPP}} / 2$, whereas the mode propagating inside the channel (that has the properties of a Bloch mode) exhibits an intensity modulation with a periodicity equal to $\sqrt{3} a$, i.e., the periodicity of the $\Gamma M$ symmetry axis of the crystal. From the cross-cuts shown in Fig. 2 , it is also clear that the SPP damping into the channel is stronger in the case of a focused illumination than for the collimated one. This difference can be attributed to a direct excitation of the channel mode by the incident light. Indeed, the resonance condition of a sufficiently large channel is expected to be close to that of a bare extended thin film. Thus, if the PC are excited by means of a collimated beam, the near-field intensity levels should be used carefully when studying PC properties such as losses or guiding efficiency along bent paths, for example. Note that, although the damping of the SPP inside the channel depends upon the illumi- nation conditions, the SPP field confinement inside the channel is not significantly different when using a collimated or a focused incident beam.

The cross-cuts displayed on the right part of Fig. 2 have been taken over the crystal on the collimated and focused illumination PSTM images. The SPP field intensity appears strongly damped with the propagation distance within the crystal. In analogy with a Bloch wave traveling through a conventional Bragg mirror ${ }^{20}$ at a frequency in the gap of the mirror, we assume that the SPP field is exponentially damped as it penetrates into the crystal. By fitting the experimental cross-cuts (shown on the right part of Fig. 2) with exponential functions, we found that the $e^{-1}$ damping distance of the SPP inside the crystal is 3.9 and $4.2 \mu \mathrm{m}$, respectively, for the collimated and focused illumination. Thus, we conclude that, when the SPP propagation through the PC is prohibited, the SPP damping distance inside the crystal is not significantly changed by the illumination conditions. In the following, we will then characterize the optical response of the PC by measuring the inner crystal SPP damping distance in the case of a collimated illumination.

\section{EXPERIMENTAL CHARACTERIZATION OF THE PC GAPS}

The physical origin of a BG in a PC being the multiple in-plane scattering, the optical properties of $\mathrm{PC}$ are expected to depend upon the size, the shape, and/or the refractive index of the surface scatterers. In this section, we investigate the PC properties as a function of the radius of the gold hemiellipsoid comprising the crystal. Because the height of the bumps may have a dramatic effect on the PC spectral properties, ${ }^{17}$ for all the samples considered in the following the bump height is kept constant and equal to $50 \mathrm{~nm}$. Changing the radius of these bumps corresponds to a change of the so-called filling factor, i.e., the ratio of the surface occupied by the number of scatterers per unit lattice cell to the surface of a unit lattice cell. For a triangular bigrating with a period $a$ and half a scatterer per unit cell, the filling factor $f$ is given by

$$
f=\frac{2 \pi R^{2}}{a^{2} \sqrt{3}}
$$

if $R$ denotes the radius of the hemiellipsoids. The goal of this work is not to demonstrate the existence of a full BG so that we restrict ourselves to SPP propagation along only two directions aligned with the main symmetry axes $\Gamma K$ and $\Gamma M$.

\section{A. Propagation along the $\Gamma K$ axis}

Figures 3(a) and 3(b) show typical PSTM images recorded over a PC with a period $a=430 \mathrm{~nm}$ and comprised of gold bumps with a diameter of $162 \mathrm{~nm}$ (filling factor of $13 \%)$. Line defects oriented along the $\Gamma K$ axis are opened into the crystals. The PC is illuminated by a collimated beam that launches a SPP on the bare gold thin-film area located on the right side of line $x=10 \mu \mathrm{m}$ on both images (a) and (b). This SPP propagates parallel to the longitudinal axis of the line defects, i.e., along the $\Gamma K$ direction. As in the case of 

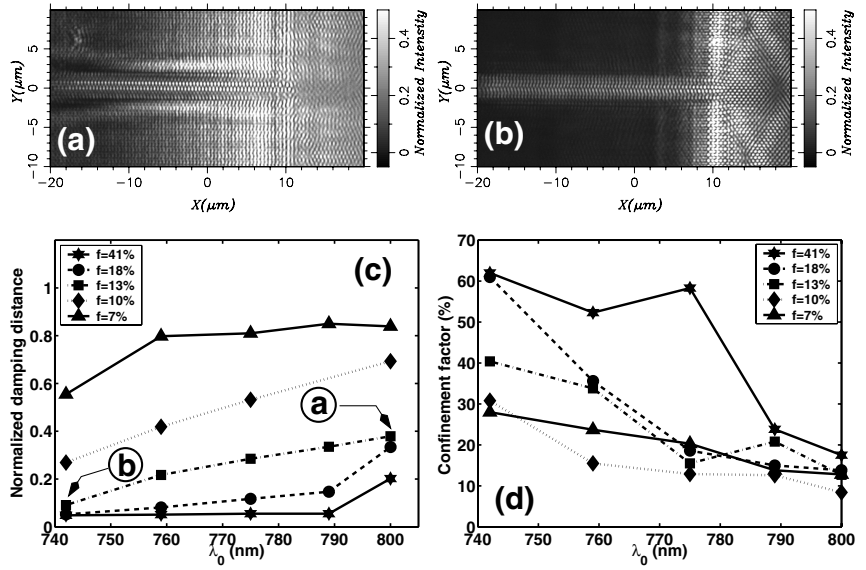

FIG. 3. PSTM images of a $\Gamma K$ oriented crystal with a period of $430 \mathrm{~nm}$ and a filling factor of 13\%. Image (a) [image (b)] has been obtained with an incident free-space wavelength of $\lambda_{0}=800 \mathrm{~nm}$ $(742 \mathrm{~nm})$. (c) Normalized damping distance of the incident SPP inside the crystal as a function of the frequency and the filling factor. The damping distance is normalized with respect to the damping distance of a thin-film SPP computed from the dielectric function tabulated in Ref. 18 The points corresponding to images (a) and (b) are shown on the curve obtained for a filling factor of $13 \%$. (d) Confinement factor of the SPP field inside the channel as a function of the filling factor and frequency. The confinement is computed according to Eq. (5) by using a transverse (i.e., perpendicular to the channel axis) cross-cut of the corresponding PSTM image obtained by averaging the near-field intensity over a strip with a width of $2 \mu \mathrm{m}$ located at a distance of $10 \mu \mathrm{m}$ from the border of the crystal.

Fig. 2, the incident SPP is traveling from the right to the left of the images. At a frequency corresponding to a free-space wavelength of $\lambda_{0}=800 \mathrm{~nm}$, the incident SPP penetrates deeply into the crystal [Fig. 3(a)], whereas on the contrary, a dramatic damping occurs at $\lambda_{0}=742 \mathrm{~nm}$. By using the procedure described in the previous section, the damping distance $d_{e}$ in the crystal is found to be $20.3 \mu \mathrm{m}(4.8 \mu \mathrm{m})$ at $\lambda_{0}=800 \mathrm{~nm}\left(\lambda_{0}=742 \mathrm{~nm}\right)$. With the aim of comparing the PC attenuation lengths measured at different frequencies, $d_{e}$ must be normalized by the $e^{-1}$ damping distance of a SPP propagating at the surface of a bare thin film. The normalized damping distance $d_{e}^{(n)}$ obtained for different frequencies and different filling factors is plotted in Fig. 3(c). For a given filling factor, the damping distance decreases monotonically with the incident free-space wavelength. At a fixed frequency, $d_{e}^{(n)}$ decreases as the filling factor increases such that the smallest values of $d_{e}^{(n)}$ are obtained for large filling factors and the small wavelengths of the frequency range we consider. In addition, it can be seen that for a given frequency, the decrease of the damping distance is larger for small values of $f$. Indeed, for $f$ changing from $18 \%$ to $41 \%$, the normalized damping distance for short wavelengths decreases only very little. The dip in the dispersion of $d_{e}^{(n)}$ observed for short wavelengths is expected to be related to a BG since for a $430 \mathrm{~nm}$-period triangular PC, the center gap wavelength is around $\lambda_{0}=680 \mathrm{~nm}$ (see Sec. II), i.e., in the blue side of our laser frequency range. However, small values of $d_{e}^{(n)}$ are not characteristic of a BG as they could also be related to the excitation of SPP modes with a short lifetime and/or a small group velocity. The existence of a BG must be checked against other parameters such as SPP field confinement in the channels or the in-plane PC reflectivity. Figure 3(d) shows the confinement factor $C$ computed according to

$$
C=\frac{\int_{-L / 2}^{+L / 2} I\left(x_{0}, y\right) d y}{\int_{-W / 2}^{+W / 2} I\left(x_{0}, y\right) d y},
$$

where $L$ and $W$ denote, respectively, the width of the channel and the scan size in the $y$ direction of the PSTM images and where $I$ is the near-field intensity measured with the PSTM. The cross-cuts of the PSTM images used to perform the calculations of $C$ have been taken at a distance of $10 \mu \mathrm{m}$ from the right border of the PC (i.e., $x_{0}=0.0 \mu \mathrm{m}$ ). The evolution of the confinement factor as a function of the frequency confirms the existence of a BG in the low-wavelength range since $C$ increases significantly with decreasing incident wavelengths. In addition, we found that increasing the filling factor improves the SPP field confinement inside the channel. Based on these experimental results, we conclude that a BG exists probably for the $\Gamma K$ direction in the blue side of our laser frequency range. This result will be checked against numerical calculations later (Sec. V). Because we observe only the long-wavelength side of this BG, we cannot conclude whether the BG width is increased or whether the central gap frequency is redshifted by increasing $f$, however we observe an evolution of the BG as a function of $f$ in qualitative agreement with the results discussed in Refs. 11 and 17

\section{B. Propagation along the $\Gamma M$ axis}

We consider now PCs featuring channels oriented along the $\Gamma M$ symmetry axis. As in the previous case, the crystals are illuminated by an incident SPP traveling parallel to the channels axis. Figures 4(a) and 4(b) show PSTM images recorded over a $\Gamma M$ oriented PC with a filling factor of $10 \%$ (bumps diameter $=140 \mathrm{~nm}$, period $a=430 \mathrm{~nm}$ ). Figure 4(a) has been obtained at a frequency corresponding to $\lambda_{0}$ $=742 \mathrm{~nm}$. Unlike what has been observed for the $\Gamma K$ direction, the incident SPP penetrates far into the crystal at this frequency. The image shown in Fig. 4(b) has been obtained by switching the incident wavelength to $\lambda_{0}=789 \mathrm{~nm}$. This time, the penetration depth is reduced and the SPP field is efficiently confined into the channel. On the basis of the PSTM images recorded at different frequencies for samples with increasing filling factors, the curves plotted in Figs. 4(c) and 4(d) have been obtained by measuring, respectively, the normalized damping distance $d_{e}^{(n)}$ and the confinement factor. For all the considered filling factors, the normalized damping distance is found to be minimum around $\lambda_{0}=780 \mathrm{~nm}$. The central gap wavelength of the $\Gamma M$ direction is then just $20 \mathrm{~nm}$ larger than the rough estimate obtained from the Bragg condition in Sec. II. For increasing filling factors, the damping distances at the long- and short-wavelength side of the BG are strongly reduced whereas the central gap wave- 

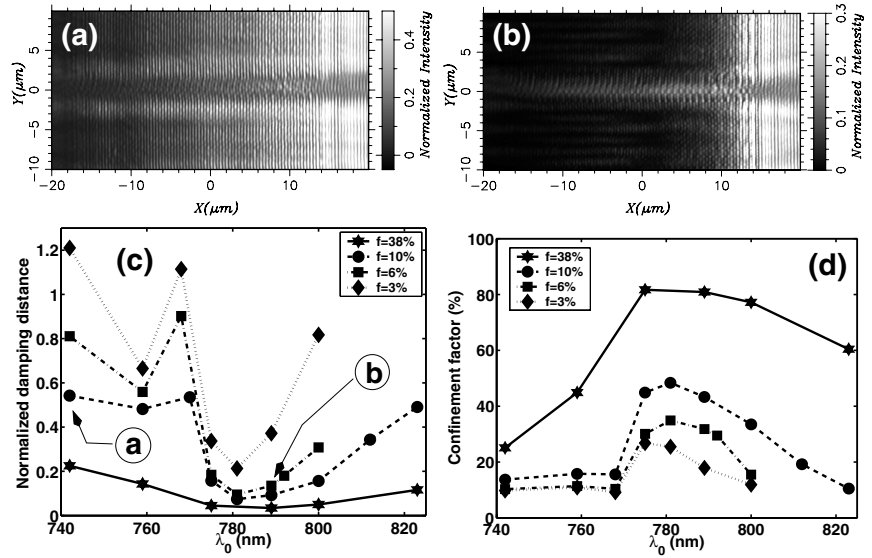

FIG. 4. PSTM images of a $\Gamma M$ oriented crystal with a period of $430 \mathrm{~nm}$ and a filling factor of $10 \%$. Image (a) [image (b)] has been obtained with an incident free-space wavelength of $\lambda_{0}=742 \mathrm{~nm}$ $(789 \mathrm{~nm})$. (c) Normalized damping distance of the incident SPP inside the crystal as a function of the frequency and the filling factor. The points corresponding to images (a) and (b) are shown on the curve obtained for a filling factor of $13 \%$. (d) Confinement factor of the SPP field inside the channel as a function of the filling factor and frequency.

length is slightly redshifted. From these observations we conclude that, in agreement with previous theoretical works, the increase of the PC filling factor enlarges the BG and redshifts the central gap frequency. ${ }^{9,16,17}$ Finally, it is worth noting that for all filling factors, the smallest inner crystal SPP penetration depth corresponds to the largest confinement factor, indicating that the BG can also be identified from the dispersion of the confinement factor. This important observation elucidates the issue of correlation between the BG position and the dispersion of SPP propagation loss and field intensity confinement for a PC channel that has recently been brought to the fore. ${ }^{17}$

\section{SPP in-plane scattering}

Up to now, the optical properties of the PC have been studied from the field distributions measured inside the crystals or the line defects. However, the PC could be characterized as well by considering the field reflected by the crystals. ${ }^{13}$ The images shown in Figs. 5(a) and 5(b) have been recorded over crystals featuring channels oriented, respectively, along the $\Gamma K$ and $\Gamma M$ directions. For both orientations, the filling factor and the incident wavelength correspond to the shortest inner crystal penetration depth, i.e., $\lambda_{0}=742 \mathrm{~nm}$ and $f=41 \%$ for $\Gamma K$ and $\lambda_{0}=775 \mathrm{~nm}$ and $f$ $=38 \%$ for $\Gamma M$. Beside the short SPP propagation inside the crystal and the efficient SPP confinement in the channels, a very well defined standing-wave pattern can be seen on both images. Although a detailed discussion of the line-defect waveguides modal structure is beyond the scope of this work (a complete study devoted to this topic will be reported elsewhere), it is, however, worthwhile to note that very different field distributions are observed in the waveguides in Figs. 5(a) and 5(b), revealing different guiding conditions for the $\Gamma K$ and $\Gamma M$ oriented channels.
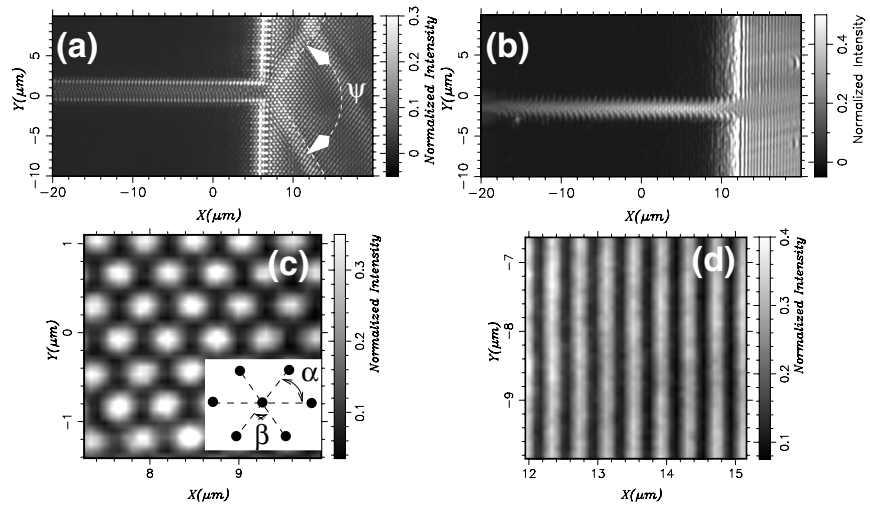

FIG. 5. (a) PSTM image of a crystal (filling factor 41\%) featuring a $\Gamma K$ oriented channel. The incident frequency corresponds to $\lambda_{0}=742 \mathrm{~nm}$. The two beams emerging from the channel form an angle $\psi=(123 \pm 5)^{\circ}$. (b) PSTM image of a crystal with a filling factor of $38 \%$ and featuring a $\Gamma M$ oriented channel. The incident frequency corresponds to $\lambda_{0}=775 \mathrm{~nm}$. (c) $[(\mathrm{d})]$ Zoom of the quasihexagonal standing wave pattern visible on image (a). The angles $\alpha$ and $\beta$ (see inset) are, respectively, equal to $54^{\circ}$ and $72^{\circ}$. (d) Zoom of the $1 \mathrm{D}$ standing-wave pattern visible on image (b).

As shown on the zooms of Figs. 5(c) and 5(d), the standing-wave pattern exhibits a quasihexagonal symmetry for the crystal with a $\Gamma K$ oriented channel as the angles $\alpha$ and $\beta$ [see the inset in Fig. 5(c)] formed by the lattice lines (of the standing-wave pattern) are, respectively, equal to $54^{\circ}$ and $72^{\circ}$, whereas a simple $1 \mathrm{D}$ pattern is obtained for the crystal with a $\Gamma M$ oriented channel. Moreover, for the $\Gamma K$ orientation [Fig. 5(a)], two beams emerging from the channel and forming an angle $\psi=(123 \pm 5)^{\circ}$ are also clearly visible [a third beam located in the upper right part of the image is also visible and is due to a line-defect waveguide located over the channel shown on Fig. 5(a)].

We believe that the in-plane scattering of the incident SPP on the $\Gamma M$ and $\Gamma K$ oriented PC boundaries is at the origin of, respectively, the quasihexagonal standing-wave pattern and the beams emerging from the channel visible in Fig. 5(a). Indeed, as shown in Fig. 6(a), for this orientation of the crystal, the incident SPP is normally incident on the $Г M$ oriented boundary of the PC, whereas it propagates parallel to the $\Gamma K$ PC boundaries comprising the edges of the linedefect waveguide. From the conservation of the momentum parallel to the PC boundary, it is a simple matter to show that a SPP normally incident on the Г $M$ PC boundary is in-plane scattered in propagation directions defined by the polar angle $\theta_{\Gamma M}[$ see Fig. 6(b)] given by

$$
\sin \theta_{\Gamma M}(m)=m \frac{\lambda_{\mathrm{SPP}}}{\sqrt{3} a},
$$

where $m$ is an integer and where $\lambda_{\mathrm{SPP}}$ and $a$ denote, respectively, the incident SPP wavelength and the period of the triangular crystal. For an incident SPP wavelength such that $\frac{\sqrt{3} a}{2}<\lambda_{\mathrm{SPP}} \leqslant \sqrt{3} a$, then only three back-reflected SPPs propagating along directions labeled as $\vec{k}_{\mathrm{SPP}}^{R}(+1), \vec{k}_{\mathrm{SPP}}^{R}(0)$ and $\vec{k}_{\mathrm{SPP}}^{R}(-1)$ in Fig. 6(b) are obtained. The angle $\theta_{\Gamma M}$ formed by the specular reflection $\vec{k}_{\mathrm{SPP}}^{R}(0)$ and the two other scattered 


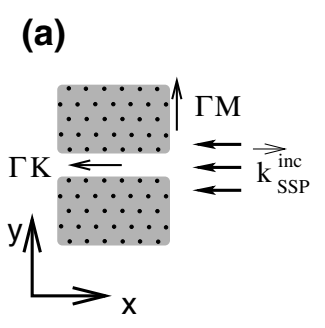

(c)

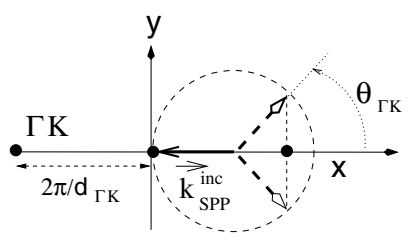

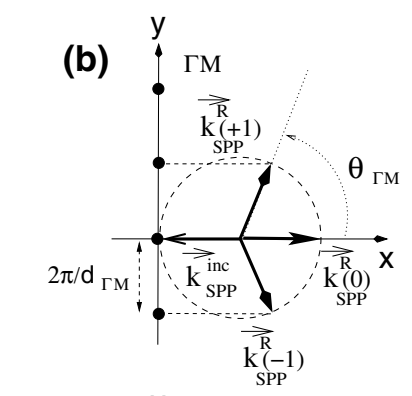

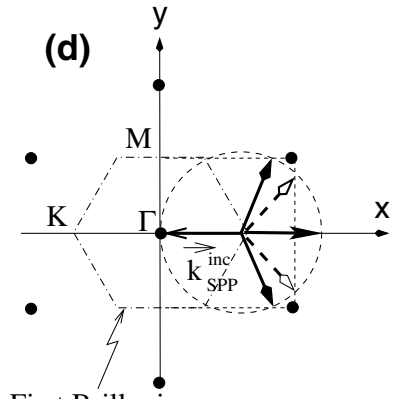

First Brillouin zone

FIG. 6. (a) Schematic view of the crystal featuring a $\Gamma K$ oriented line-defect waveguide. (b) In-plane (elastic) scattering diagram of the incident SPP on the $\Gamma M$ interface of the PC. The period of the $1 \mathrm{D}$ grating comprising the $\Gamma M$ oriented PC interface is $d_{\Gamma M}=\sqrt{3} a$ if $a$ denotes the period of the triangular crystal. (c) Inplane scattering diagram of the incident SPP on the $\Gamma K$ oriented PC interfaces. The period of the grating is $d_{\Gamma K}=a$. (d) Ewald construction showing simultaneously the in-plane backscattered directions of the incident SPP on the $\Gamma M$ (solid arrows) and $\Gamma K$ (dashed arrows) oriented PC interfaces. The in-plane scattering directions are given by the crossing of the isofrequency contour of the incident SPP (circle with a radius $\left|k_{\text {SPP }}\right|$ ) with the normal to the PC interfaces passing through the vertices of the triangular reciprocal lattice.

orders $(+1)$ and $(-1)$ is equal to $60^{\circ}$ for the incident frequency equal to the Bragg reflected frequency of the $\Gamma K$ direction.

At the entrance of the channel, the incident SPP hits at grazing incidence the line-defect waveguide boundaries comprised of gratings with a period $a$ leading to in-plane scattering directions given by [see Fig. 6(c)]

$$
\cos \theta_{\Gamma K}(m)=-1+m \frac{\lambda_{\mathrm{SPP}}}{a},
$$

where $m$ is a positive integer. As shown in Fig. 6(c), only two scattering directions corresponding to $m=1$ are obtained for $\frac{a}{2}<\lambda_{\mathrm{SPP}} \leqslant a$. As in the previous case, for an incident frequency equal to the $\Gamma K$ Bragg reflected frequency, the angle $\theta_{\Gamma K}(1)$ is equal to $60^{\circ}$. However, as shown in Fig. 6(d), if the incident wavelength is slightly larger than the $\Gamma K$ Bragg reflected wavelength, then the corresponding $\theta_{\Gamma M}$ and $\theta_{\Gamma K}$ angles are, respectively, larger and smaller than $60^{\circ}$.

The quasihexagonal interference pattern observed in Figs. 5(a) and 5(c) can now be easily interpreted as the result of the interaction of three surface waves. Indeed, having in mind that the 1D interference pattern of two SPPs is oriented parallel to the median axis formed by their wave vectors, the quasihexagonal interference pattern observed in the experi-

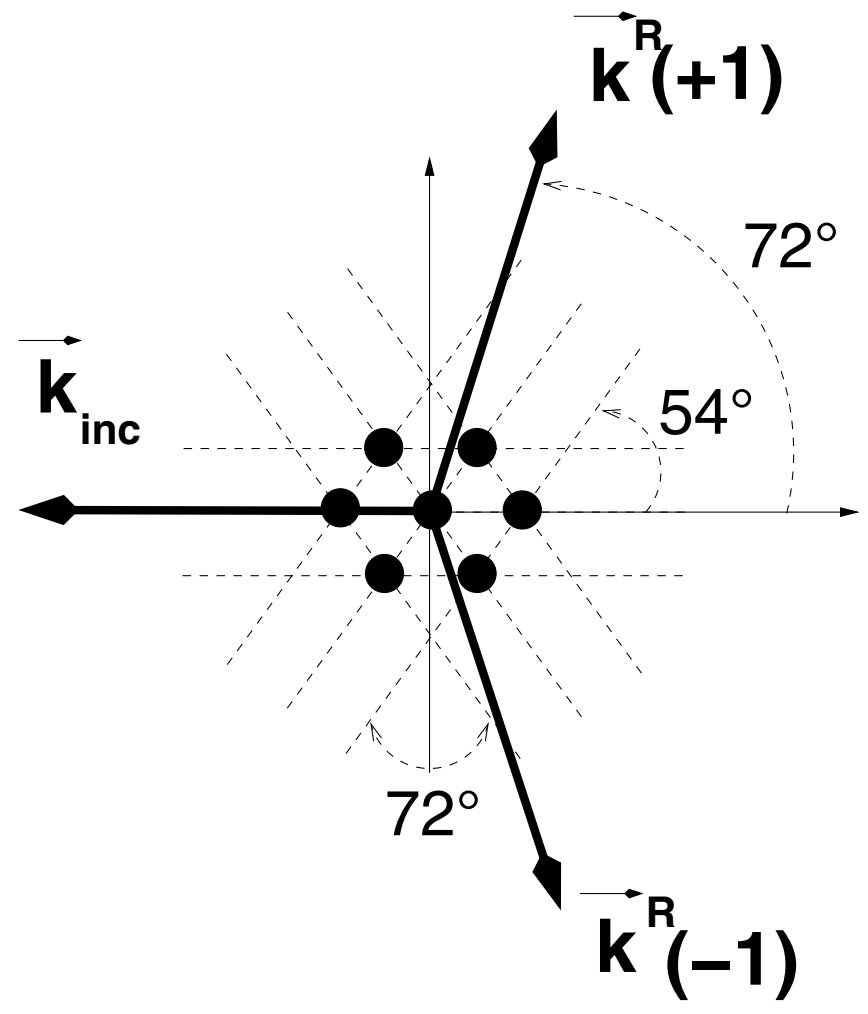

FIG. 7. Schematic view of the origin of the quasihexagonal standing-wave pattern observed on the experimental images (see Fig. 5). The orientation of the lattice lines of the standing-wave pattern fits with the experimental results if the backscattered orders propagate along a direction forming an angle $\theta_{\Gamma M}=72^{\circ}$.

mental images can be understood as the superposition of the 1D interference fringes created by the mutual interaction of the incident SPP with the two orders $(+1)$ and $(-1)$ backscattered by the $\Gamma M$ oriented PC interface. As shown in Fig. 7, the symmetry of the experimental quasihexagonal standingwave pattern is restored if we assume a scattering angle $\theta_{\Gamma M}(1)$ for the backscattered orders of $72^{\circ}$. The scattering angle $\theta_{\Gamma M}(1)$ computed from Eq. (6) for a free-space wavelength of $742 \mathrm{~nm}\left(\lambda_{\mathrm{SPP}}=722 \mathrm{~nm}\right)$ and a lattice constant $a$ $=430 \mathrm{~nm}$ leads to a value of $76^{\circ}$, in fairly good quantitative agreement with the experimental value. Note that our interpretation of the standing-wave pattern holds only if the amplitude of the specularly back-reflected order (0) is negligible or at least small compared to the amplitude of the $(+1)$ and $(-1)$ scattered orders.

Let us consider now the scattering of the incident SPP on the very first periods of the $\Gamma K$ oriented PC boundaries comprising the edges of the line-defect waveguides. As mentioned before, the two diverging beams emerging from the channel are attributed to this scattering mechanism. However, when computing from Eq. (7) the full opening angle formed by the two scattered orders for conditions that correspond to the experimental situation, we end up with a value of roughly $\psi=95^{\circ}$, significantly different from the experimental one $(\psi=123 \pm 5)^{\circ}$. The origin of this discrepancy is still not fully understood, however it could be just due to the fact that we estimate the scattering angle by using a grating 
(a)
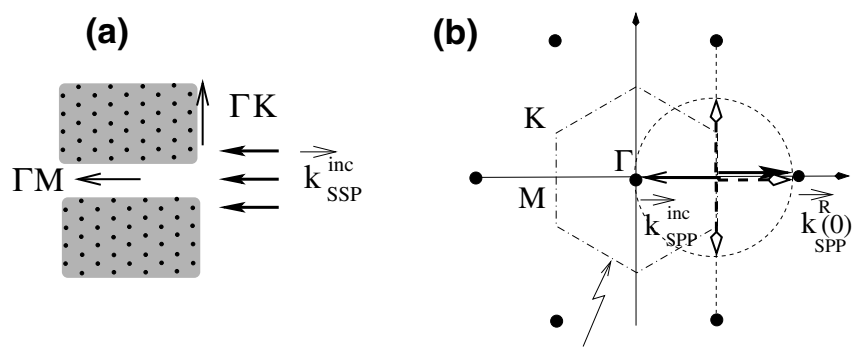

First Brillouin zone
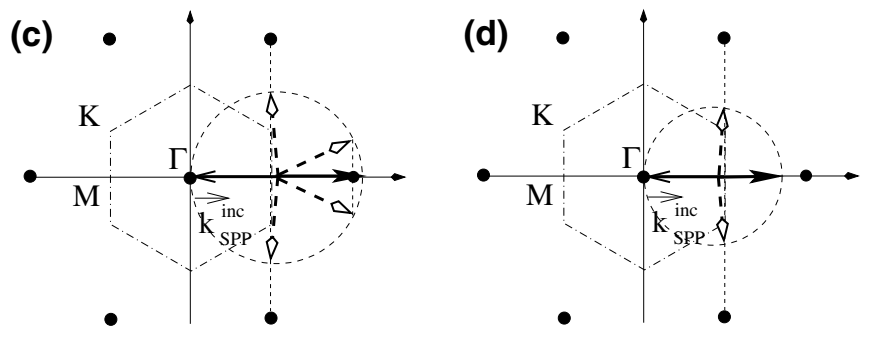

FIG. 8. (a) Schematic view of the crystal featuring a $\Gamma M$ oriented channel. (b) Ewald construction showing backscattered SPP scattering directions on the $\Gamma K$ (solid arrow) and $\Gamma M$ oriented PC interfaces. The incident SPP wavelength corresponds to the Bragg wavelength of the crystal $\Gamma M$ axis. Note that the first Brillouin zone has been rotated $30^{\circ}$ with respect to Fig. $6(\mathrm{~d})$ in order to align the $\Gamma M$ axis with the actual incident SPP propagation direction on the near-field images. (c) [(d)] Same as (b) except that the incident SPP wavelength is smaller (larger) than the $\Gamma M$ axis Bragg wavelength.

formula whereas probably only few periods of the line-defect waveguide edges contribute to this beam generation.

Our interpretation of the beam origin is, however, supported by the results obtained for a crystal with line-defect waveguides oriented along the $\Gamma M$ direction [Figs. 5(b) and 5(d)]. As shown in Fig. 8, the incident SPP is normally incident on the $\Gamma K$ oriented PC interface leading to a single back-reflected specular scattered order (solid arrow) that generates the 1D standing-wave pattern observed on the experimental images. On the other hand, the incident SPP falling at grazing incidence on the two $\Gamma M$ oriented edges of the channel generates in-plane scattered orders with propagation directions that depend on the incident wavelength. Indeed, for an incident SPP wavelength matching the $Г M$ Bragg wavelength [Fig. 8(b)], the scattering on the edges of the channel leads to SPPs propagating (dashed arrows) either perpendicular or parallel to the channel axis. For an incident SPP wavelength slightly smaller [Fig. 8(c)] than the $Г M$ Bragg wavelength, the scattered waves of first order are no longer perpendicular to the channel axis and the scattered SPPs of second order propagate with a small angle with respect to the channel axis. If the incident SPP wavelength is larger than the $\Gamma M$ Bragg wavelength [Fig. 8(d)], only firstorder scattered SPPs can exist. Thus, for an incident SPP wavelength close to the ГM Bragg wavelength, the SPPs corresponding to the first diffraction order by the edges of the channel are unable to escape from it (as they propagate almost perpendicular to the channel) whereas the secondorder SPPs (when they exist) propagate almost parallel to the channel and then parallel to the SPP back-reflected by the $\Gamma K$ oriented crystal surface. Thus, when they exist, only the

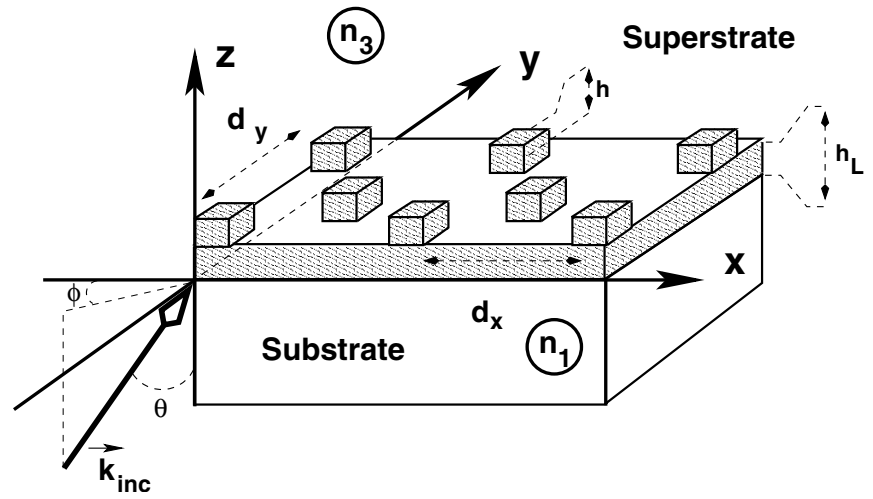

FIG. 9. Schematic view of the numerical PC. The PCs are comprised of gold parallelepipeds of golds deposited on a gold thin film. The period of the bigrating is, respectively, $d_{x}$ and $d_{y}$ along the Cartesian axis $x$ and $y$. The angle of incidence and the in-plane propagation direction of the incident monochromatic plane wave is controlled by, respectively, the angle $\theta$ and the angle $\phi$.

second-order SPPs diffracted by the few first periods of the $\Gamma M$ oriented channel boundaries could generate a beam, and in this case the beam is expected to propagate in the same direction as the SPPs strongly back-reflected by the $\Gamma K$ oriented crystal surface, making it difficult to observe such a beam in the near-field images.

At this stage, it is worth noting that the field distributions observed inside the channels are likely related to the scattering of the SPPs traveling into the channels on the gratings comprising the edges of the line-defect waveguides. As mentioned before, a detailed analysis of these field distributions will be report elsewhere, however one can easily anticipate that the concepts used to understand the standing-wave patterns outside the crystals hold also for observation points located inside the line-defect waveguides.

\section{MODELING PLASMONIC CRYSTALS}

\section{A. Plasmonic crystals band diagrams}

From an experimental point of view, the characterization of $\mathrm{PC}$ is a tedious and time-consuming task as it requires us to consider incident wave vectors distributed along the whole boundary of the irreducible Brillouin zone. In this context, the full understanding of PC properties greatly benefits from numerical modeling.

The model we use in this section is not expected to describe the surface modes sustained by a periodically modulated metallic surface more accurately than previously reported PC models, ${ }^{9,11,17}$ but it allows us to consider computational situations that are close to our experimental configuration. Specifically, the PCs we consider numerically are deposited on a metal thin film (as in our experiments) and this might have some importance as the PC properties could be influenced by the metal/superstrate SPP radiation leakage through the thin film. ${ }^{11}$ The numerical PCs are comprised of infinitely extended gratings of the gold scatterers deposited at the surface of a gold thin film [with a thickness $h_{L}$ lying on a glass substrate (see Fig. 9)]. The sample is illuminated by a 
monochromatic incident plane wave, the orientation of the incident wave vector being controlled by two angles $\theta$ and $\phi$. The metallic part of the object (thin film and scatterers) is described by their complex dielectric function tabulated in Ref. 18 such that we account for the Ohmic losses of gold. The formalism we use to study these PCs is known as the differential method. ${ }^{21,22}$ When dealing with bigratings, the differential method relies on a truncated $2 \mathrm{D}$ plane-wave expansion of the electromagnetic field,

$$
\vec{A}(x, y, z)=\sum_{m=-M}^{+M} \sum_{n=-N}^{+N} \vec{A}(m, n) \exp \left[i\left(\alpha_{m} x+\beta_{n} y+\gamma_{m, n} z\right)\right],
$$

where $\vec{A}$ denotes the electric or magnetic field. The wavevector components of a plane wave in expansion (6) are given by

$$
\begin{gathered}
\alpha_{m}=n_{1} k_{0} \sin \theta \cos \phi+m \frac{2 \pi}{d_{x}}, \\
\beta_{n}=n_{1} k_{0} \sin \theta \sin \phi+n \frac{2 \pi}{d_{y}}, \\
\left(\gamma_{m, n}^{(l)}\right)^{2}=\left(n_{l} k_{0}\right)^{2}-\alpha_{m}^{2}-\beta_{n}^{2},
\end{gathered}
$$

where $n_{1}$ is the refractive index of the substrate. The superscript (l) in Eq. (9) can be either "1" or " 3 " depending whether the field is considered in the substrate or in the superstrate. From Eq. (9), it is clear that the $z$ wave-vector component can be real or pure imaginary introducing, respectively, radiative or evanescent orders in the expansion (8).

The use of the differential method has been restricted for a long time to the study of dielectric gratings due to numerical instabilities when dealing with metallic objects. These instabilities have been found to be due to (i) numerical contamination related to the existence of evanescent orders in the field expansion and (ii) a poor convergence of this field expansion with the number of orders involved in the truncated series. About a decade ago, the explanation of the poor field expansion convergence was found ${ }^{23}$ and solutions have been proposed. ${ }^{24,25}$ Based on these solutions, stable algorithms have been developed among which is the " $S$-matrix algorithm" used in this work. A detailed description of this algorithm can be found in Ref. 21. In order to keep the acceleration procedure of the field expansion convergence ${ }^{26}$ [denoted as the fast Fourier factorization (FFF) method in Ref. 21] at a reasonable level of complexity, all the numerical results discussed in the following have been obtained using PCs comprised of periodically distributed scatterers with vertical sidewalls, i.e., parallelepipeds.

For a given incident field $\left(\vec{E}^{\text {inc }}, \vec{H}^{\text {inc }}\right)$, the $S$-matrix algorithm returns the values of the plane-wave amplitudes $\vec{A}(m, n)$ for the field in the substrate or in the superstrate. Knowing the field amplitudes in the substrate, it is a simple matter to compute the reflectivity of the sample at the incident frequency according to

$$
R=\sum_{(m, n) \mathrm{rad}} e_{m, n}^{(1)},
$$

where $e_{m, n}^{(1)}$ is the efficiency of the plane wave labeled by the two integers $(m, n)$ and where the sum is taken over all radiative plane waves in the substrate. For a given plane wave $(m, n)$, the efficiency is obtained from

$$
e_{m, n}^{(1)}=\frac{\vec{S}_{m, n}^{(1)} \cdot \vec{u}_{z}}{\vec{S}^{\text {inc }} \cdot \vec{u}_{z}},
$$

where $\vec{S}_{m, n}^{(1)}$ and $\vec{S}^{\text {inc }}$ are, respectively, the Poynting vector of the $(m, n)$ and incident [i.e., $(0,0)]$ plane waves. The SPP modes sustained by the periodically modulated thin films can be characterized by computing the sample reflectivity for different incident frequencies and different illumination conditions. Note that in this way, we adopt the same technique as that used in Ref. 4 to demonstrate experimentally the existence of a full band gap in a PC. At a given incident wavelength $\lambda_{0}$, the wave-vector component parallel to the sample surface can be adjusted by changing the angle of incidence $\theta$ while choosing convenient $\phi$ allows us to investigate SPP excitation along different directions. Thus the dispersion curves of the SPP modes sustained by the PC can be obtained from the computation of the textured surface reflectivity.

The PCs we consider numerically are comprised of triangular bigrating of parallelepipeds with a volume $h \times L^{2}$, where $h$ is the height of the scatterers and $L$ is the lateral size of their square basis. Our implementation of the differential method can only deal with bigratings that are periodic along the $x$ and $y$ directions of the Cartesian basis, therefore the triangular PCs are defined from a rectangular unit cell shown in Figs. 10(a) and 10(c). This numerical unit lattice cell contains two scatterers and has a surface of $a^{2} \sqrt{3}$ if $a$ is the period of the triangular lattice. The filling factor of the numerical PC is then given by

$$
f=\frac{2 L^{2}}{a^{2} \sqrt{3}} .
$$

The shape of the numerical scatterers (parallelepipeds) differs significantly from that of the experimental surface defects (hemiellipsoids) such that we restrict ourselves to the study of numerical PCs with a quite low filling factor. Indeed, for high $f$, the top surface of the parallelepipeds is equivalent to a metal surface perturbed by air holes whereas the surface of hemiellipsoids, even when closely packed, is still very rough. Thus, the SPP modes sustained by numerical PCs with high filling factors are not expected to be similar to that of the experimental crystals. The reflectivity maps displayed in Figs. 10(b) and 10(d) have been obtained for PC with a filling factor of, respectively, $0.6 \%$ and $28 \%[L$ $=30 \mathrm{~nm}(L=215 \mathrm{~nm}), a=430 \mathrm{~nm}, h=50 \mathrm{~nm}]$. These maps have been computed by using 196 plane waves in the electromagnetic field expansion for frequencies standing in the visible and near-infrared and propagation directions sweeping the boundary of the irreducible Brillouin zone $\Gamma K(\phi$ $\left.=0^{\circ}\right), \Gamma M\left(\phi=30^{\circ}\right)$, and $M K$. On these maps, the dark areas, which correspond to low reflectivity, characterize the PC 


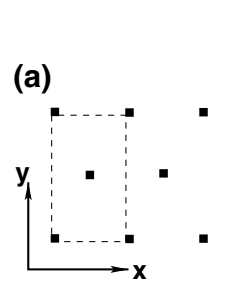

(b)
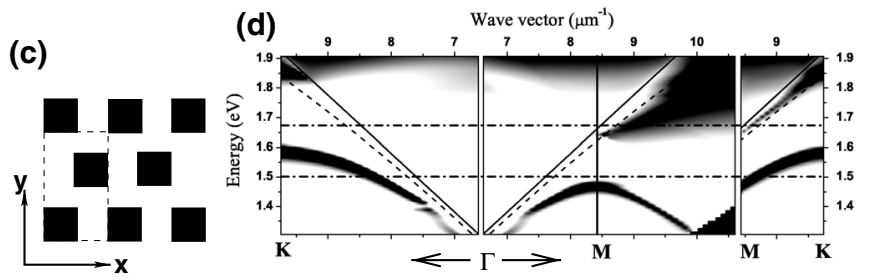

FIG. 10. (a) and (c) Top view of two numerical PC with a period of $430 \mathrm{~nm}$ and a filling factor of, respectively, $0.6 \%$ and $28 \%$. (b) and (d) Reflectivity maps computed for the PC with, respectively, $f=0.6 \%$ and $28 \%$. To increase the contrast of the low reflectivity areas, the color scale is ranging from $R=80 \%$ (black) to $R=90 \%$ (white). The dark areas are related to large absorption and thus correspond to SPP mode excitation. On each diagram, the solid lines show the light line of air, the dashed line is the dispersion curve of a flat thin-film SPP, and the dash-dotted lines are the limits of our laser frequency range. The symmetry points defining the irreducible Brillouin zone are shown on each diagram. Note that the $\Gamma$ point is not reached. On these plots, the abscissa is the in-plane wave-vector component of the incident plane wave for each part of the irreducible Brillouin zone boundary including for the MK segment.

SPP modes excitation. Experimentally, we have observed the near-field response of the crystals illuminated by flat thinfilm SPPs with frequencies distributed into our laser range. The computed dispersion curve of the flat thin-film SPP is shown as a dashed line on the reflectivity maps. For the $\Gamma M$ direction, the computation of the textured thin-film reflectivity has been pushed beyond the limit of the first Brillouin zone because for frequencies in the short-wavelength part of our laser range, the flat thin-film SPP excitation is achieved for wave vectors that are larger than the wave vector corresponding to the high-symmetry point $M$. Note that, for the $\Gamma M$ direction, the illumination conditions defined by the points located inside the dark triangles in the lower right corner of the maps cannot be treated with our model as they correspond to evanescent waves in the glass substrate.

For a filling factor of $0.6 \%$, narrow energy band gaps open up at the boundary of the first Brillouin zone. At the high-symmetry point $K$, the gap occurring around $1.77 \mathrm{eV}$ $\left(\lambda_{0}=700 \mathrm{~nm}\right)$ is on the short-wavelength side of our laser range, whereas at $M$ the gap is within this range and centered around $1.58 \mathrm{eV}\left(\lambda_{0}=784 \mathrm{~nm}\right)$. These results are in agreement with our experimental observations, in particular the theoretical center gap frequency in the $\Gamma M$ direction is only few nanometers redshifted with respect to the experimental one $\left(\lambda_{0}=780 \mathrm{~nm}\right.$ for the smallest experimental filling factor $f=7 \%$ ). We note that, according to these calculations, a PC with $f=0.6 \%$ does not create a full gap in the dispersion curve of the SPP modes. If the filling factor is increased to $28 \%$, the BGs are clearly enlarged in all propagation direc- tions creating a full $\mathrm{BG}$ around $1.62 \mathrm{eV}(764 \mathrm{~nm})$. At $K$, the gap is now about $0.2 \mathrm{eV}$ wide and centered around $1.71 \mathrm{eV}$ $\left(\lambda_{0}=724 \mathrm{~nm}\right)$. From the analysis of the experimental data obtained for the $\Gamma K$ oriented crystals, we could not conclude whether the increase of the filling factor enlarges the gap or redshifts the central gap frequency. This calculation shows that although a redshift of the central frequency occurs, this is mainly the BG enlargement that explains the redshift of the long-wavelength BG edge observed experimentally for increasing $f$. Similarly, at the symmetry point $M$, the increase of $f$ redshifts only very little the center frequency, whereas a significant BG enlargement occurs. The central gap wavelength $(790 \mathrm{~nm})$ is found to be one more time in good agreement with the experimental central frequency observed for the highest filling factors (around $790 \mathrm{~nm}$ for $f=38 \%$ ). The experimental short-wavelength BG edge (around $770 \mathrm{~nm}$ ) seems to be slightly redshifted when compared to the theoretical one $(750 \mathrm{~nm})$. Concerning the long-wavelength edge, the experimental value seems to be out of our laser range, in agreement with the value of $826 \mathrm{~nm}$ obtained numerically. At this point it should be noted, however, that an accurate estimate of the BG width is difficult to obtain from our experimental data not only because of the limited frequency range of our laser but also because the set of $\left(\omega, k_{\|}\right)$points considered experimentally are distributed along the dispersion curve of a thin-film SPP [shown as dashed lines in Figs. 10(b) and 10(d)] and thus does not allow us to investigate the whole range of wave vectors necessary to observe entirely the $\mathrm{BG}$, as was done, for example, in Ref. 4.

\section{B. PC in-plane reflectivity}

We have seen previously that a finite-size PC can reflect efficiently an incident SPP in directions corresponding to inplane scattering provided that the incident frequency belongs to the crystal band gap. Therefore, the in-plane (or modal) reflectivity is an interesting parameter as it characterizes the PC band gap without ambiguity. In principle, the measurement of the PC modal reflectivity is possible by considering the depth of modulation of the standing-wave pattern observed in the PSTM images [see Figs. 5(c) and 5(d)]. However, this depth of modulation being dependent on the quality of the tip used to record the PSTM images, only relative values of the modal reflectivity for different incident frequencies can be obtained in this way. With the aim of characterizing the in-plane scattering efficiency of PC as a function of the filling factor, we compute in this last paragraph the modal reflectivity of semi-infinite PC comprised of gold scatterers with different shapes. As in the experiment, we consider that a SPP launched on a flat gold interface is incident onto a semi-infinite PC region with a given orientation $\Gamma K$ or $\Gamma M$ [see Fig. 11(a)]. The geometric parameters used for the computation are the following: the PC is assumed to be composed of a triangular array of bumps with a lattice period $a=430 \mathrm{~nm}$ and the bumps have either a cylindrical or a hemi-ellipsoidal shapes with a 50-nm height [see Fig. 11(b)]. Gold is considered as a real metal with a finite conductivity and its frequency-dependent permittivity is taken from Ref. 18. The incident SPP is supposed to be excited at 

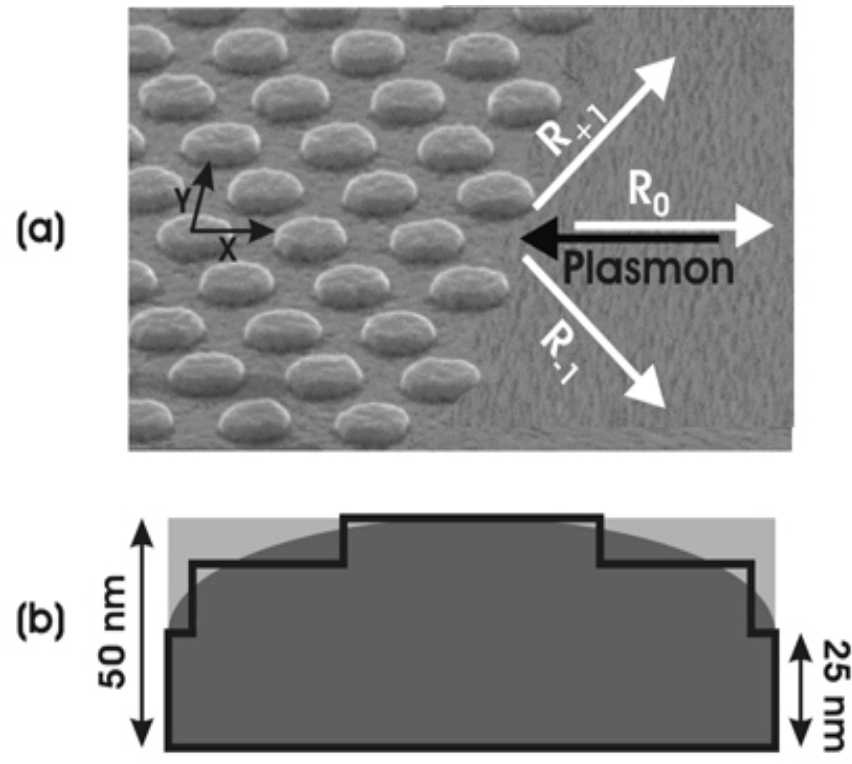

FIG. 11. Definition of the physical and numerical parameters used for the computational results of Fig. 9. (a) SPP scattering problem. The depicted situation corresponds to a SPP normally incident from the $\Gamma K$ direction. (b) Staircase approximation of the hemiellipsoidal bumps used for the calculation. Light gray shadow: cylindrical bump. Dark gray shadow: actual hemi-ellipsoidal bump. Black curve: staircase approximation.

the interface between two semi-infinite gold/air half-spaces. Although this situation departs from the experimental one (a 50 -nm-thick gold film is used in the experiment for the purpose of the Kretschmann-Raether SPP excitation), it allows us to compute the intrinsic (or optimum) modal reflectivity of the PC as the use of a bulk metal interface prevents leakages from the SPP radiation into the dielectric substrate supporting the gold thin film. The 3D frequency-domain modal method used for the computation is described in Ref. 27. In brief, the method relies on an analytical integration of Maxwell's equations along the incident direction and on a supercell approach in the two others. Periodic boundary conditions are used in the $y$ direction and perfectly matched layers implemented as nonlinear coordinate transforms ${ }^{28,29}$ are used in the $z$ direction to carefully handle out-of-plane far-field radiations in the air clad. Since these layers absorb nonevanescent radiations, the electromagnetic fields are null on the $z$ boundaries of the supercell and are thus periodic functions of the $z$ coordinates. This allows the calculation of the radiated and guided modes in a Fourier (plane-wave) basis in each layer (the hemi-ellipsoidal bumps are discretized in a series of thin uniform layers in the incident direction) and the integration in the incident direction by relating recursively the mode amplitudes in the different layers using a scattering matrix approach. Convergence tests have been performed to estimate the accuracy of the computational results by increasing the truncation rank $N$ of the electromagnetic field Fourier expansions in the transverse $y$ and $z$ directions from $N= \pm 1$ to $N=35$ (i.e., 5041 plane waves). Stable results for the modal reflectivity have been obtained for $N>16$, letting us expect an absolute error of 0.01 on the calculated data. Thus the only approximation results from the staircase pro-
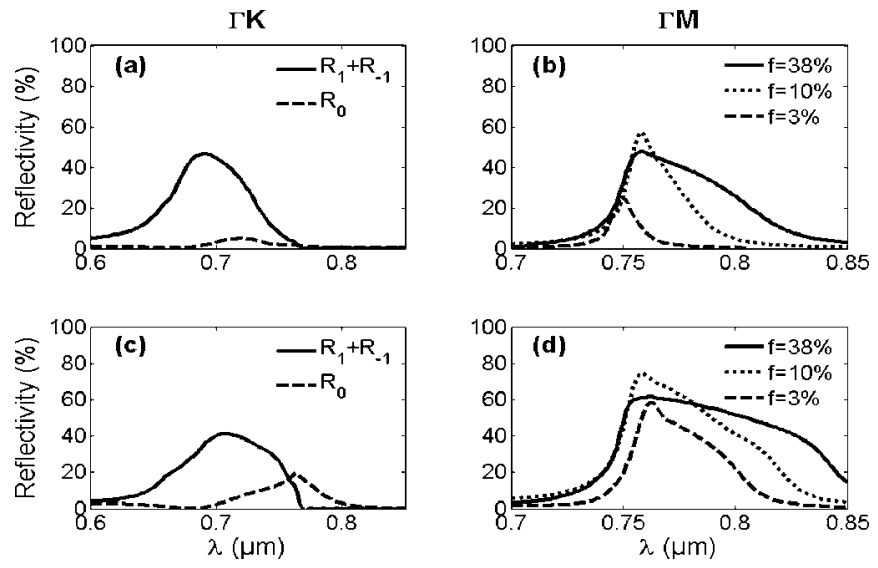

FIG. 12. Computational results for the SPP modal reflectivity for the $\Gamma M\left(R_{0}\right)$ and $\Gamma K\left(R_{0}\right.$ and $\left.R_{-1}+R_{+1}\right)$ directions. (a) and (b) are relative to the hemi-ellipsoidal shapes actually fabricated. (c) and (d) are relative to ideal cylindrical shapes. In (a) and (c) the filling factor is $f=41 \%$.

file used to numerically encode the hemi-ellipsoid [see Fig. 11(b)]. The computed data have also been cross-checked with other available data. For instance, a quantitative agreement has been obtained with the computational results of Fig. 1 in Ref. 17 although the two methods are totally different from a numerical point of view.

Figures 12(a) and 12(b) show the modal reflectivity computed over a broad spectral interval in the case of an incident SPP traveling, respectively, along the $\Gamma K$ or $\Gamma M$ directions. For the $\Gamma K$ direction, the total modal reflectivity is found to be the sum of the specular reflectivity denoted by $R_{0}$ hereafter and the positive $\left(R_{1}\right)$ and negative $\left(R_{-1}\right)$ first-order scattered SPPs [see Fig. 11(a)]. The curves displayed in Fig. 12(a) have been obtained for a filling factor of $41 \%$ and hemi-ellipsoidal scatterers. The calculation confirms the existence of a band gap centered around $\lambda=680 \mathrm{~nm}$, a wavelength out of the tunability range of the titanium-sapphire laser used in the experiment. Moreover, we note that the reflection mostly occurs in the negative and positive firstorder $\left(R_{1}+R_{-1}\right.$ reaches approximatively $50 \%$ with $R_{-1}=R_{+1}$ for symmetry reasons) whereas the specular reflectivity remains rather low $\left(R_{0}<7 \%\right)$. This fully explains the hexagonal near-field pattern of Fig. 5(c). For an incident SPP traveling along the $\Gamma M$ direction, the SPP reflection occurs only in the specular direction. Therefore, the curves plotted in Fig. 12(b) show the specular reflectivity $R_{0}$ computed for PCs with different filling factors and composed of hemiellipsoidal scatterers. Although the central gap frequency of the PC with a small filling factor is blueshifted with respect to the experimental results (by about 5\%), the computational results strongly support the experimental trends. On the one hand, the diminution observed experimentally in the inner crystal penetration depth is due to an increase of the reflectivity and not to some additional losses in the corrugated metal. On the other hand, the broadening of the band gap highly depends on the filling factor of the hemi-ellipsoidal bumps. Nevertheless, for large filling factors this broadening is achieved at the expense of the maximal reflectivity, which is higher for $f=10 \%$ than for $f=38 \%$. Therefore, a compro- 
mise has to be found between the band-gap width and the maximal reflectivity. It should also be noted that the spectral evolution of the modal reflectivity is very similar to that of the confinement factors displayed in Fig. 4(d). In particular, the short-wavelength band-gap edge is found to be more abrupt than the long-wavelength one on both the experimental plots of the confinement factor and the modal reflectivity curves obtained for the $\Gamma M$ oriented crystals. This behavior can be attributed to the appearance of an out-of-plane scattered order for wavelengths that are shorter than the central gap wavelength. Indeed, the appearance of an out-of-plane channel for the SPP scattering necessarily reduced the inplane SPP scattering efficiency and thus the modal reflectivity of the PC. Finally, we stress another important issue, namely the shape of the bumps. Up to now, we have considered hemi-ellipsoidal bumps as close as possible to the actual bump shapes. If we consider perfectly cylindrical bumps [see Fig. 11(b)], the results are rather different, as illustrated in Figs. 12(c) and 12(d). A direct visual comparison with Figs. 12(a) and 12(b) evidences that for both directions, the band gap is wider and the reflectivity higher for the cylinders than for the hemi-ellipsoids. Indeed, a small change of the bump profile can deeply modify the properties of the PC, which shows the importance of the fabrication process.

\section{CONCLUSION}

In summary, we have operated a PSTM to study the influence of the filling factor on the optical properties of plasmonic crystals comprised of finite-size triangular gratings of gold bumps deposited on a gold thin film. By considering either the penetration length of an incident thin-film SPP into the crystals or the SPP field confinement inside a line defect open through the crystals, we have found that changing the filling factor of a PC can dramatically modify its properties. In particular, we have demonstrated experimentally the bandgap enlargement for increasing values of the filling factor in the two high-symmetry propagation directions $\Gamma M$ and $\Gamma K$. These results have been further confirmed by computing with the differential method the reflectivity of metallic thin films textured by triangular lattices of gold parallelepipeds. Based on these reflectivity computations, we have plotted the dispersion curves of the SPP modes sustained by PC. These SPP dispersion curves exhibit the expected energy BG at the boundary of the irreducible Brillouin zone. In agreement with our experimental results, these gaps are enlarged by an increase of the filling factor, and a moderate redshift of the central gap frequency occurs in the $\Gamma K$ direction. From both numerical and experimental results, we conclude that a careful choice of the filling factor is necessary to optimize the performances of PC. In particular, by choosing the correct filling factor, an overlap of the BG in several propagation directions can be obtained leading to an efficient SPP field confinement into line defects oriented along these directions and, eventually, to low loss SPP propagation, bending and splitting with these defects. Note, however, that such a multidirectional efficient SPP field confinement may still turn out to be not sufficient to achieve high SPP transmission along bent line defects. Indeed, a vanishing density of surface modes at a given frequency for different directions does not prevent from the losses related to inelastic-scattering process and then a short lifetime of line defect SPP modes. Finally, we would like to emphasize that there exist other more important and fascinating applications of PC structures than those related to guiding (bending, splitting, etc.) of radiation. One can, for example, employ SPP propagation along PC channels near the BG edges for significantly slowing down the SPP propagation, similar to what has been recently accomplished with conventional photonic crystal waveguides, ${ }^{30}$ a phenomenon that exhibits rich physics and can be advantageously exploited for increasing efficiency of nonlinear optical interactions adding up to the contribution from the SPP related field enhancement.

\section{ACKNOWLEDGMENTS}

This work was supported by the European Network of Excellence (NoE) Plasmo-Nano-Devices, the French Ministry of Education and Research, and partially by the NoE NEMO. The authors acknowledge Jean-Paul Hugonin for fruitful discussions and computational helps.
${ }^{1}$ H. Raether, Surface Plasmons, Springer Tracts in Modern Physics Vol. 111 (Springer, Berlin, 1988).

${ }^{2}$ D. Egorov, B. S. Dennis, G. Blumberg, and M. I. Haftel, Phys. Rev. B 70, 033404 (2004).

${ }^{3}$ W. L. Barnes, T. W. Preist, S. C. Kitson, J. R. Sambles, N. P. K. Cotter, and D. J. Nash, Phys. Rev. B 51, 11164 (1995).

${ }^{4}$ S. C. Kitson, W. L. Barnes, and J. R. Sambles, Phys. Rev. Lett. 77, 2670 (1996).

${ }^{5}$ W. L. Barnes, T. W. Preist, S. C. Kitson, and J. R. Sambles, Phys. Rev. B 54, 6227 (1996).

${ }^{6}$ H. Ditlbacher, J. R. Krenn, G. Schider, A. Leitner, and F. R. Aussenegg, Appl. Phys. Lett. 81, 1762 (2002).

${ }^{7}$ J.-C. Weeber, Y. Lacroute, A. Dereux, E. Devaux, T. Ebbesen, C. Girard, M. U. González, and A.-L. Baudrion, Phys. Rev. B 70,
235406 (2004).

${ }^{8}$ J.-C. Weeber, M. U. González, A.-L. Baudrion, and A. Dereux, Appl. Phys. Lett. 87, 221101 (2005).

${ }^{9}$ M. Kretschmann and A. A. Maradudin, Phys. Rev. B 66, 245408 (2002).

${ }^{10}$ M. Notomi, Phys. Rev. B 62, 10696 (2000).

${ }^{11}$ M. Kretschmann, Phys. Rev. B 68, 125419 (2003).

${ }^{12}$ J. D. Joanopoulos, R. D. Meade, and J. N. Winn, Photonic Crystals (Princeton University Press, Princeton, NJ, 1995).

${ }^{13}$ S. I. Bozhevolnyi, J. Erland, K. Leosson, P. M. W. Skovgaard, and J. M. Hvam, Phys. Rev. Lett. 86, 3008 (2001).

${ }^{14}$ S. I. Bozhevolnyi, V. S. Volkov, K. Leosson, and A. Boltasseva, Appl. Phys. Lett. 79, 1076 (2001).

${ }^{15}$ C. Marquart, S. I. Bozhevolnyi, and K. Leosson, Opt. Express 13, 
3303 (2005).

${ }^{16}$ T. Sondergaard and S. I. Bozhevolnyi, Phys. Rev. B 67, 165405 (2003).

${ }^{17}$ T. Sondergaard and S. I. Bozhevolnyi, Phys. Rev. B 71, 125429 (2005).

${ }^{18}$ Handbook of Optical Constants of Soloids, edited by D. Palik (Academic, New York, 1985).

${ }^{19}$ J-C Weeber, J. R. Krenn, A. Dereux, B. Lamprecht, Y. Lacroute, and J-P Goudonnet, Phys. Rev. B 64, 045411 (2001).

${ }^{20} \mathrm{P}$. Yeh, in Optical Waves in Layered Media, edited by J. W. Goodman, Wiley Series in Pure and Applied Optics (Wiley, New York, 1988).

${ }^{21}$ M. Nevière and E. Popov, Light Propagation in Periodic Media (Dekker, New York, 2003).
${ }^{22} \mathrm{P}$. Vincent, in Electromagnetic Theory of Gratings, edited by R. Petit (Springer-Verlag, Berlin, 1980).

${ }^{23}$ L. Li, J. Opt. Soc. Am. A 13, 1870 (1996).

${ }^{24}$ P. Lalanne and G. M. Morris, J. Opt. Soc. Am. A 13, 779 (1996).

${ }^{25}$ L. Li, J. Opt. Soc. Am. A 14, 2758 (1997).

${ }^{26}$ E. Popov and M. Neviére, J. Opt. Soc. Am. A 18, 2886 (2001).

${ }^{27}$ E. Silberstein, P. Lalanne, J. P. Hugonin, and Q. Cao, J. Opt. Soc. Am. A 18, 2865 (2001).

${ }^{28}$ J. P. Berenger, J. Comput. Phys. 114, 185 (1994).

${ }^{29}$ J. P. Hugonin and P. Lalanne, J. Opt. Soc. Am. A 22, 1844 (2005).

${ }^{30}$ Y. A. Vlasov, M. O'Boyle, H. F. Hamann, and S. J. Mc Nab, Nature (London) 438, 65 (2005). 